\title{
Macromolecular salen catalyst with largely enhanced catalytic activity
}

Susanne Striegler, ${ }^{* a, b}$ Moses G. Gichinga, Michael Dittel ${ }^{\mathrm{b}}$

${ }^{a}$ Department of Chemistry and Biochemistry, Auburn University, 179 Chemistry Building, Auburn, AL 36849, USA

${ }^{b}$ Division of Inorganic Chemistry II, University of Ulm, Albert-Einstein-Allee 11, 89069 Ulm, Germany

susanne.striegler@auburn.edu

Content:

1. Kinetic data for the transformation of DTBC into DTBQ by dinuclear copper(II) complex 4 .

2. TEM image of the polymer microbeads.

3. Isothermal titration calorimetric data for the activation of the macromolecular catalyst.

4. Experimental details and characterization data for all new compounds 
1. Kinetic data for the transformation of DTBC into DTBQ by $200 \mu \mathrm{M}$ dinuclear copper(II) complex 4 in methanol at $30^{\circ} \mathrm{C} ; \mathrm{k}_{\text {cat }}=0.04 \mathrm{~min}^{-1} ; \mathrm{K}_{\mathrm{M}}=37 \mathrm{mM}$

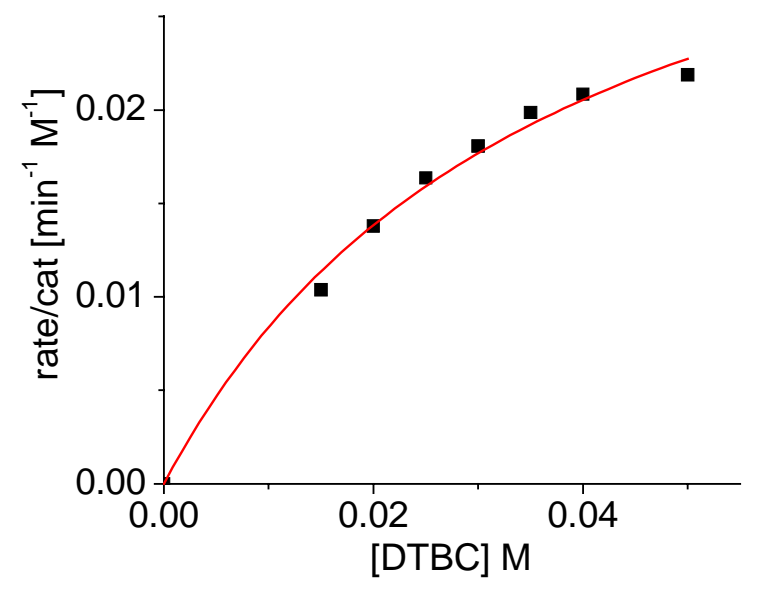

2. TEM image of ligand $\mathbf{2}$ immobilized in poly(acrylate) microbeads.

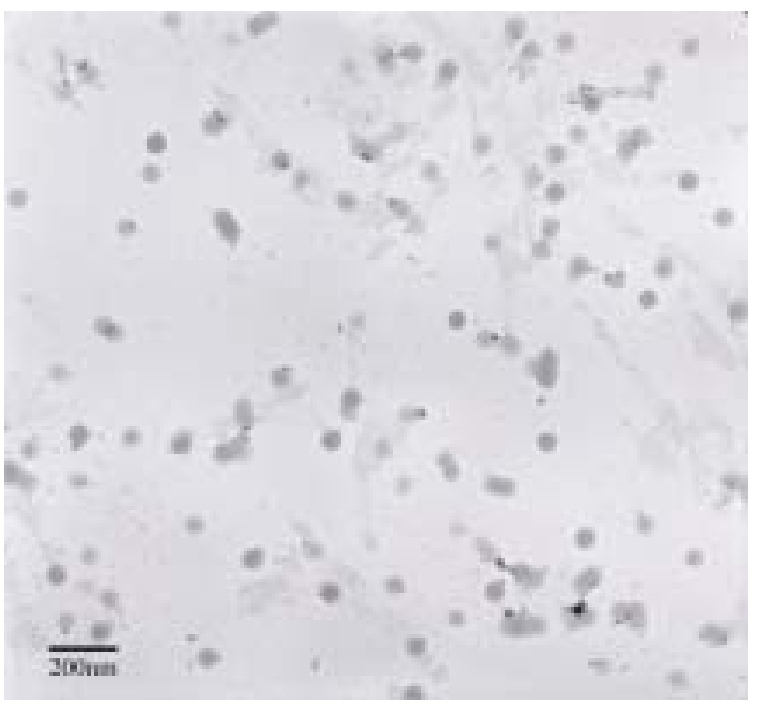


3. ITC data for activation of the polymeric catalyst by titration of the polymer with copper(II) acetate reveals complex formation with the immobilized ligand in a $2: 1$ molar ratio.

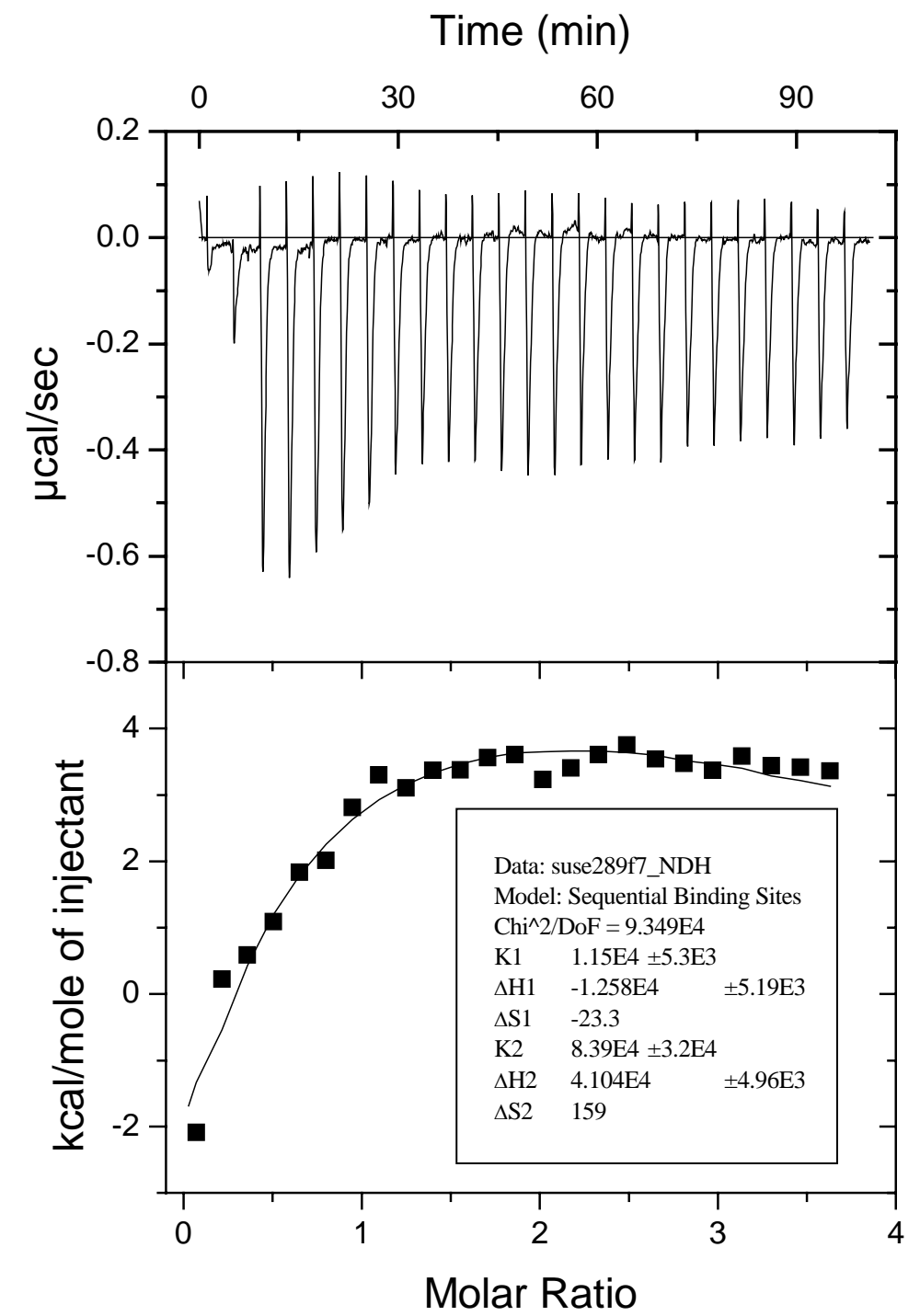

The first mol of $\mathrm{Cu}$ (II) ions is bound exothermic with a binding constant $\mathrm{K}_{1}=11500 \pm 5300$ $\left(\mathrm{pK}_{\mathrm{a}}=4\right)$ and $\Delta \mathrm{H}_{1}=-12580 \pm 5190 \mathrm{kcal} \mathrm{mol}^{-1}, \Delta \mathrm{S}_{1}=-23.3 \mathrm{cal} \mathrm{mol}^{-1} \mathrm{~K}^{-1}$ into the pentadentate salen ligand, whereas the second mold of $\mathrm{Cu}$ (II) ions binds endothermic one order of magnitude stronger with a binding constant $\mathrm{K}_{2}=83900 \pm 3200\left(\mathrm{pK}_{2}=4.9\right)$ and $\Delta \mathrm{H}_{2}=41040 \pm 4960 \mathrm{kcal}$ $\mathrm{mol}^{-1}, \Delta \mathrm{S}_{1}=159 \mathrm{cal} \mathrm{mol}^{-1} \mathrm{~K}^{-1}$. 


\section{Experimental details}

All solvents and reagents were reagent grade quality or better and were used without further purification unless stated otherwise. Methanol, ethanol, ethyl acetate, dichloromethane, acetonitrile, sodium acetate, potassium iodide were obtained from VWR International; 1,3diaminopropan-2-ol, copper(II) acetate monohydrate, copper(II) perchlorate hexahydrate, $p$ vinylbenzylchloride were purchased from Aldrich; potassium carbonate, 3,5-di-tert.butylcatechol and 2,4-dihydroxybenzaldehyde were obtained from Fluka.

The melting points are uncorrected and were measured with a Büchi B-540. The IR spectra were obtained on a Bruker IFS $133 \mathrm{~V}, v$ in $\mathrm{cm}^{-1}$, samples as $\mathrm{KBr}$ pellets. The microanalysis was performed on an Elementar Vario EL. The EI MS and FAB-MS spectra were obtained on a Finnigan MAT SSQ 7000. The NMR experiments were performed on a Bruker DRX $400\left({ }^{1} \mathrm{H}\right.$ : $400.1 \mathrm{MHz} ;{ }^{13} \mathrm{C}: 100.6 \mathrm{MHz}$ ) at $300 \mathrm{~K}, \mathrm{CDCl}_{3}$ was used as solvent and residual $\mathrm{CHCl}_{3}$ was used as internal standard $\left(\mathrm{CHCl}_{3}: \delta\left({ }^{1} \mathrm{H}\right) 7.24 ; \delta\left({ }^{13} \mathrm{C}\right) 77.0\right)$. Chemical shifts $(\delta)$ are expressed in ppm downfield from tetramethylsilane using the residual non-deuterated solvent as standard. The electronic spectra were recorded on a J \& M TIDAS UV spectrometer (software SPECTRALYS version 1.55) or Varian Cary 50 with Suprasil ${ }^{\circledR}$ standard cuvettes $(200-2000 \mathrm{~nm}$, thickness 10 $\mathrm{mm}$ and $700 \mu \mathrm{L}-3000 \mu \mathrm{L}$ volume) at $25^{\circ} \mathrm{C}$ and $30^{\circ} \mathrm{C}$. Absorbances were measured from 200 to $900 \mathrm{~nm}$.

\section{2-hydroxy-4-[(4-vinylbenzyl)oxy]benzaldehyde 1.}

A suspension of $10.15 \mathrm{~g}(0.0735 \mathrm{~mol})$ 2,4-dihydroxybenzaldehyde, $10.15 \mathrm{~g}(0.0735 \mathrm{~mol})$ potassium carbonate, $3.68 \mathrm{~g}(0.0222 \mathrm{~mol})$ potassium iodide and $15 \mathrm{~mL}(0.0954 \mathrm{~mol}) p$ vinylbenzyl chloride was stirred in $150 \mathrm{~mL}$ acetonitrile at $60^{\circ} \mathrm{C}$ for $24 \mathrm{~h}$. Evaporation of the solvents yielded a red residue, which was dissolved in a mixture of $25 \mathrm{~mL}$ of water containing $1.2 \mathrm{~mL}$ concentrated hydrochloride acid and $50 \mathrm{~mL}$ ethyl acetate. After separation of the organic layer, the aqueous phase was extracted with $15 \mathrm{~mL}$ ethyl acetate. The combined organic phases were washed two times with $20 \mathrm{~mL}$ of $3 \%$ aqueous potassium carbonate solution, $20 \mathrm{~mL}$ of water and $20 \mathrm{~mL}$ of $5 \%$ citric acid. Evaporation of the solvent results in a crude product, which was recrystallized from ethyl acetate yielding 1 as a crystalline colourless solid (13.3 g, 71\%); mp 97-99 ${ }^{\circ} \mathrm{C}$; Found: C, 75.8; H, 5.8. $\mathrm{C}_{16} \mathrm{H}_{14} \mathrm{O}_{3}$ requires C, 75.6; H, 5.6 \%; $v_{\max }(\mathrm{KBr}) / \mathrm{cm}^{-1}$ 3086, 3057, 3037, 2837, 2750, 1642, 1629, 1369, 1220; $\delta_{\mathrm{H}}\left(400 \mathrm{MHz}, \mathrm{CDCl}_{3}\right) 11.46(1 \mathrm{H}, \mathrm{s}$, phenolic $\mathrm{OH}), 9.70(1 \mathrm{H}, \mathrm{d}, \mathrm{CHO}, 0.5), 7.41(3 \mathrm{H}, \mathrm{m}), 7.35(2 \mathrm{H}, \mathrm{m}), 6.71(1 \mathrm{H}, \mathrm{dd}, 17.6,10.9,-$ $\left.\mathrm{CH}=\mathrm{CH}_{2}\right), 6.59(1 \mathrm{H}, \mathrm{dd}, 8.7,2.4), 6.49(1 \mathrm{H}, \mathrm{d}, 2.3), 5.76\left(1 \mathrm{H}, \mathrm{dd}, 17.6,0.8,-\mathrm{CH}=\mathrm{CH}_{a} \mathrm{H}_{\mathrm{b}}\right), 5.26$ $\left(1 \mathrm{H}, \mathrm{dd}, 10.9,0.8,-\mathrm{CH}=\mathrm{CH}_{\mathrm{a}} \mathrm{H}_{b}\right), 5.06\left(2 \mathrm{H}, \mathrm{s}, \mathrm{ArCH}_{2}-\mathrm{O}\right) ; \delta_{\mathrm{C}}\left(100 \mathrm{MHz}, \mathrm{CDCl}_{3}\right)$ 194.3, 165.7, $164.4,137.7,136.2,135.3,135.1,127.7,126.5,115.3,114.4,108.8,101.6,70.1 ; \mathrm{m} / \mathrm{z}$ (EI) 254 $\left(8 \%, \mathrm{M}^{+}\right), 117(100) 91(16)$.

\section{$N, N^{\prime}$-1,3-bis[(2-hydroxy-4-styryloxy)benzylideneamino]propan-2-ol 2, bssdpo.}

A solution containing $620 \mathrm{mg}(2.44 \mathrm{mmol})$ of 1 dissolved in $150 \mathrm{~mL}$ ethanol/methanol $(1: 1, \mathrm{v} / \mathrm{v})$ was added at ambient temperature to an ethanolic solution $(10 \mathrm{~mL})$ of 1,3-diaminopropan-2-ol $(110 \mathrm{mg}, 1.22 \mathrm{mmol}$ ) and allowed to react for $48 \mathrm{~h}$. Partial evaporation of the solvent gave 2 as a yellowish solid, which was purified by recrystallization from ethanol/methanol $(1 / 1, \mathrm{v} / \mathrm{v})(0.57$ g, 83\%); mp 175-177 ${ }^{\circ} \mathrm{C}$; Found: C, 74.7; H, 5.9; N, 5.0; $\mathrm{C}_{35} \mathrm{H}_{34} \mathrm{~N}_{2} \mathrm{O}_{5}$ requires $\mathrm{C}$, 74.7; H, 6.1; $\mathrm{N}, 5.0 \% ; v_{\max }(\mathrm{KBr}) / \mathrm{cm}^{-1} 3407,3086,2922,1635,1284,1220,1116\left(\mathrm{C}_{\text {aliph }}-\mathrm{O}\right) ; \delta_{\mathrm{H}}(400 \mathrm{MHz}$, $\left.\mathrm{CDCl}_{3}\right) 8.14(2 \mathrm{H}, \mathrm{s}, \mathrm{CH}=\mathrm{N}), 7.36(8 \mathrm{H}, \mathrm{dd}, 20.8,8.2, \mathrm{ArH}), 7.05(2 \mathrm{H}, \mathrm{d}, 8.4, \mathrm{ArH}), 6.70(2 \mathrm{H}, \mathrm{dd}$, 
17.4, 10.8, 2H, $\left.-\mathrm{CH}=\mathrm{CH}_{2}\right), 6.42(4 \mathrm{H}, \mathrm{m}), 5.73\left(2 \mathrm{H}, \mathrm{dd}, 17.6,0.7,-\mathrm{CH}=\mathrm{CH}_{a} \mathrm{H}_{\mathrm{b}}\right), 5.24(2 \mathrm{H}, \mathrm{dd}$, 10.8, 0.7, $\left.-\mathrm{CH}=\mathrm{CH}_{\mathrm{a}} \mathrm{H}_{b}\right), 5.02\left(4 \mathrm{H}, \mathrm{s}, \mathrm{Ar}-\mathrm{CH}_{2}\right), 4.14(1 \mathrm{H}, \mathrm{m}, \mathrm{CHOH}), 3.73(2 \mathrm{H}, \mathrm{dd}, 12.6,4.0)$, $3.60(2 \mathrm{H}, \mathrm{dd}, 12.6,6.5), 1.24(1 \mathrm{H}, \mathrm{br} \mathrm{s}, \mathrm{CHOH}) . \delta_{\mathrm{C}}\left(100 \mathrm{MHz} ; \mathrm{CDCl}_{3}\right) 166.0,165.8,163.0$, $137.5,136.4,135.9,133.0,127.7,126.4,114.2,112.4,107.2,102.3,70.3,69.7,61.5 ; \mathrm{m} / \mathrm{z}(\mathrm{FAB}$ MS) $563\left(\mathrm{MH}^{+}, 100 \%\right), 447$ (21), 327(44), 296(44) 255 (23).

\section{$\left\{N, N^{\prime}\right.$-1,3-bis[(2-hydroxy-4-styryloxy)benzylideneamino]propan-2-ol\}ato ( $\mu$-acetato)} dicopper complexes $3 \mathrm{a}, \mathrm{Cu}_{2}(\mathrm{bssdpo})(\mathrm{Ac})$

A solution of $253 \mathrm{mg}(0.45 \mathrm{mmol})$ of 2 in $25 \mathrm{~mL}$ ethanol was added dropwise to a solution of $200 \mathrm{mg}$ (1 mmol) copper(II) acetate monohydrate in methanol and refluxed for $15 \mathrm{~h}$. Evaporation of the solvents leads to a dark green/blue residue, which was purified by recrystallization from $\mathrm{CH}_{2} \mathrm{Cl}_{2} / \mathrm{MeOH}(1 / 1, \mathrm{v} / \mathrm{v})$ yielding $3 \mathbf{a}$ as a dark green/blue needles (261 mg, 78\%); mp (decomp.): 234-244 ${ }^{\circ} \mathrm{C}$; Found: C, 55.0; H, 4.7; N, 3.3; $\mathrm{C}_{37} \mathrm{H}_{34} \mathrm{~N}_{2} \mathrm{O}_{7} \mathrm{Cu}_{2} \bullet \mathrm{CH}_{2} \mathrm{Cl}_{2}$ requires: $\mathrm{C}, 54.9 ; \mathrm{H}, 4.3 ; \mathrm{N}, 3.4 \% ; v_{\max }(\mathrm{KBr}) / \mathrm{cm}^{-1}: 3051,2912,1635,1610,1568,1527,1423$, $1123\left(\mathrm{C}_{\text {aliph }}-\mathrm{O}\right) ; m / z$ (FAB MS) $745\left(\mathrm{M}^{+}, 20 \%\right) 686$ (45), 570(18), 420 (100).

\section{$\left\{N, N^{\prime}\right.$-bis[(4-styryloxy-salicyl)methylene]-1,3-diaminopropan-2-olato $\}$ ( $\mu$-benzoato)} dicopper(II) complex 3b, $\mathrm{Cu}_{2}$ (bssdpo)(benzoato)

A methanol $(200 \mathrm{~mL})$ solution of $2(1000 \mathrm{mg}, 1.78 \mathrm{mmol})$ and of potassium hydroxide $(281 \mathrm{mg}$, $5.02 \mathrm{mmol}$ ) was added to a methanol solution of copper(II) benzoate ( $845 \mathrm{mg}, 2.77 \mathrm{mmol}$ ) Slow evaporation of the solvent at ambient temperature resulted in dark green/blue crystals after several weeks (646 mg, $45 \%$ ); $\mathrm{mp}$ (decomp.) $271-281{ }^{\circ} \mathrm{C}$; Found: C, 58.6; H, 4.8; N, 3.1. $\mathrm{C}_{42} \mathrm{H}_{36} \mathrm{~N}_{2} \mathrm{O}_{7} \mathrm{Cu}_{2} \bullet 3 \mathrm{H}_{2} \mathrm{O}$ requires: C, 58.5; H, 4.9; N, 3.3; $v_{\max }(\mathrm{KBr}) / \mathrm{cm}^{-1}: 3063,2930,1638$, 1609, 1590, 1549, 1525, $1123\left(\mathrm{C}_{\text {aliph }} \mathrm{O}\right) ; \mathrm{m} / z$ (FAB MS) 807 (20\%, M+), 686 (65), 570 (22), 420 (100). 


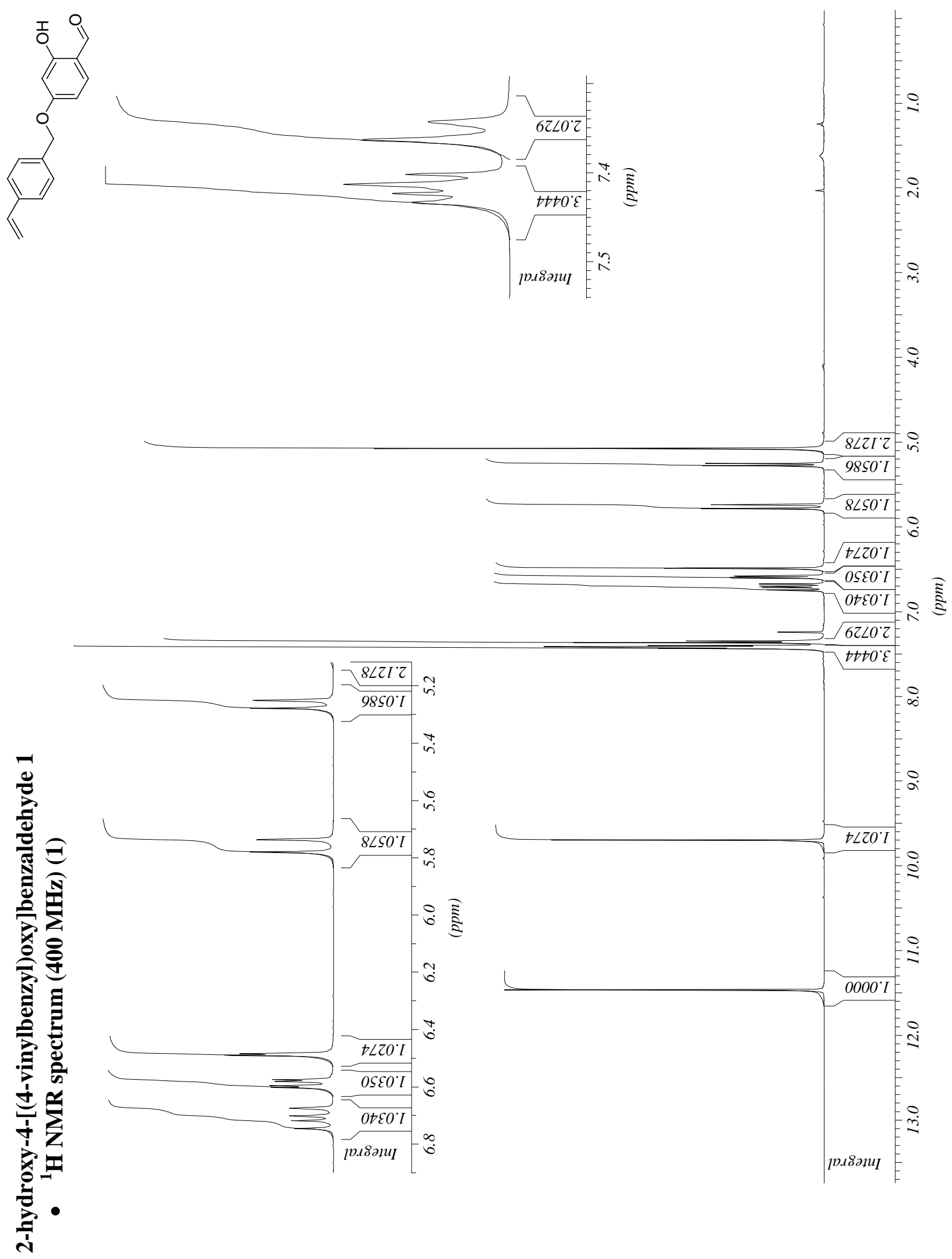



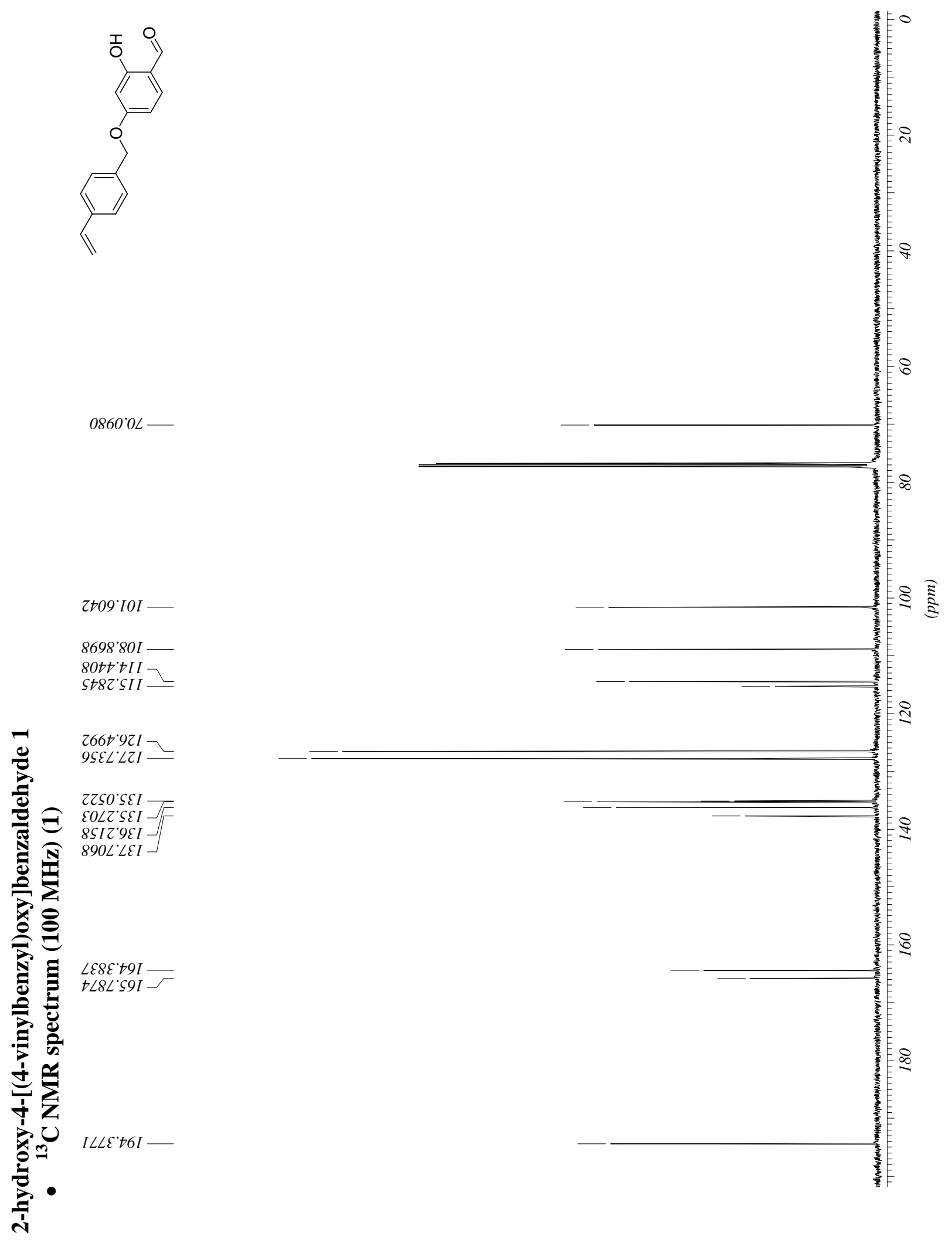

v 


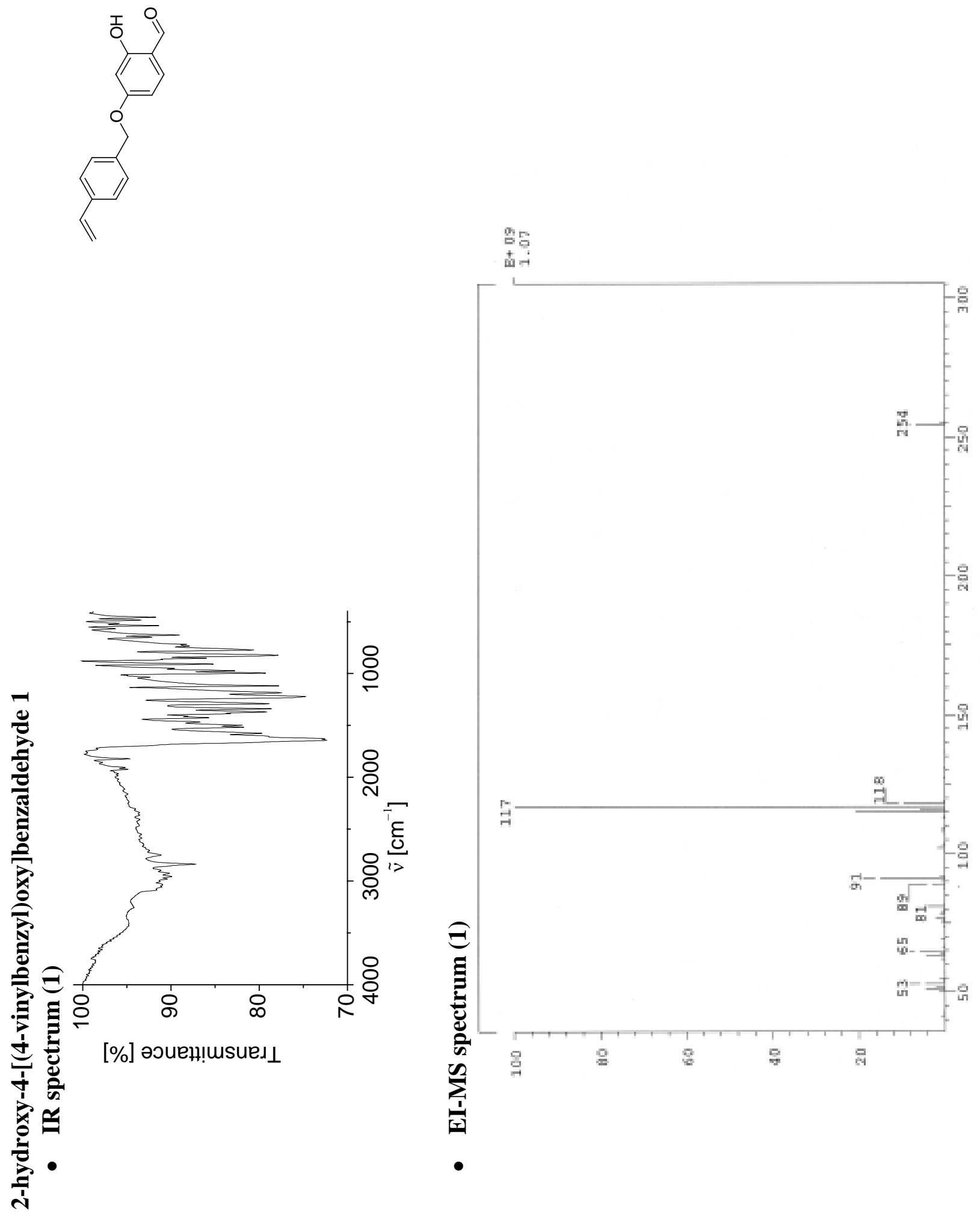




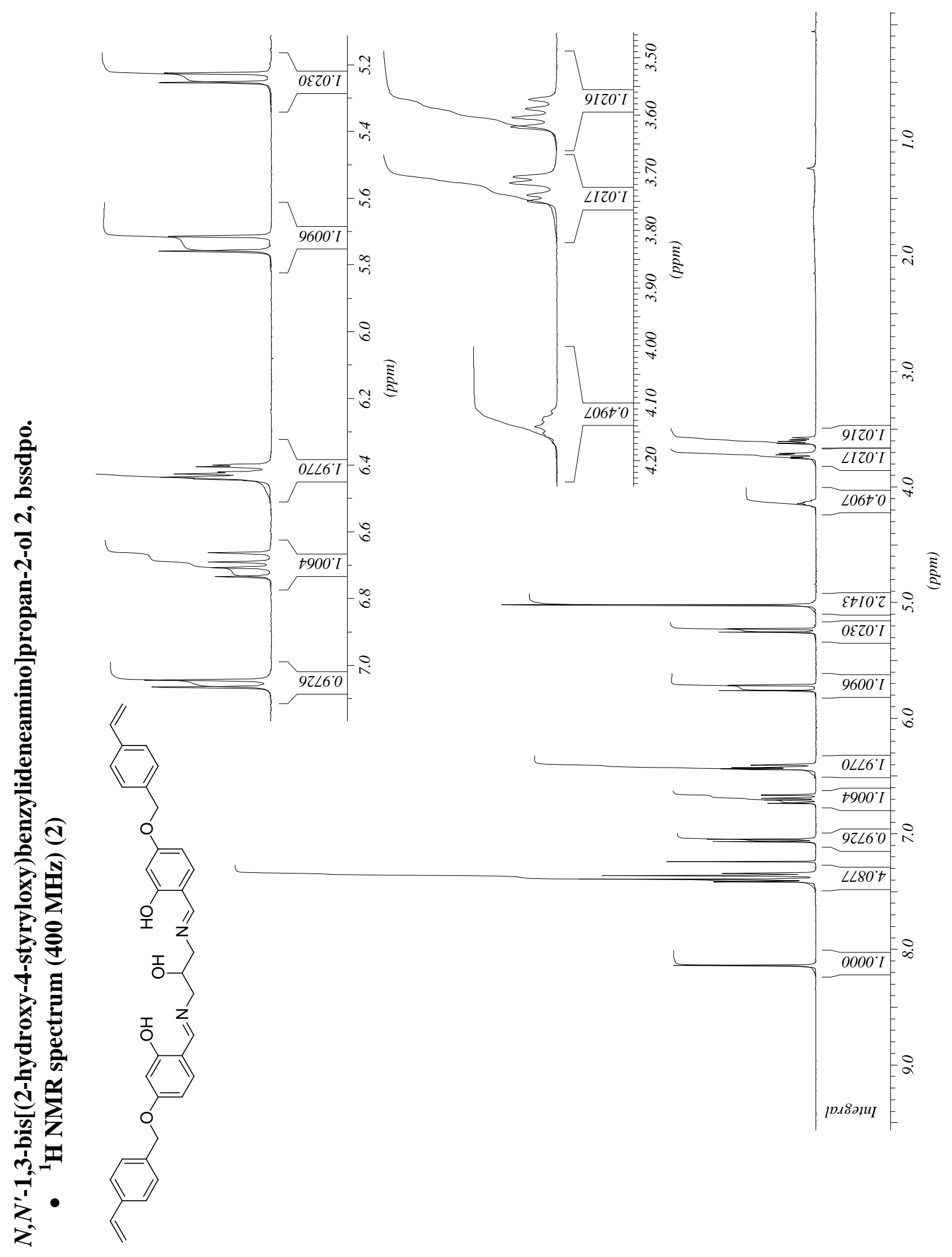




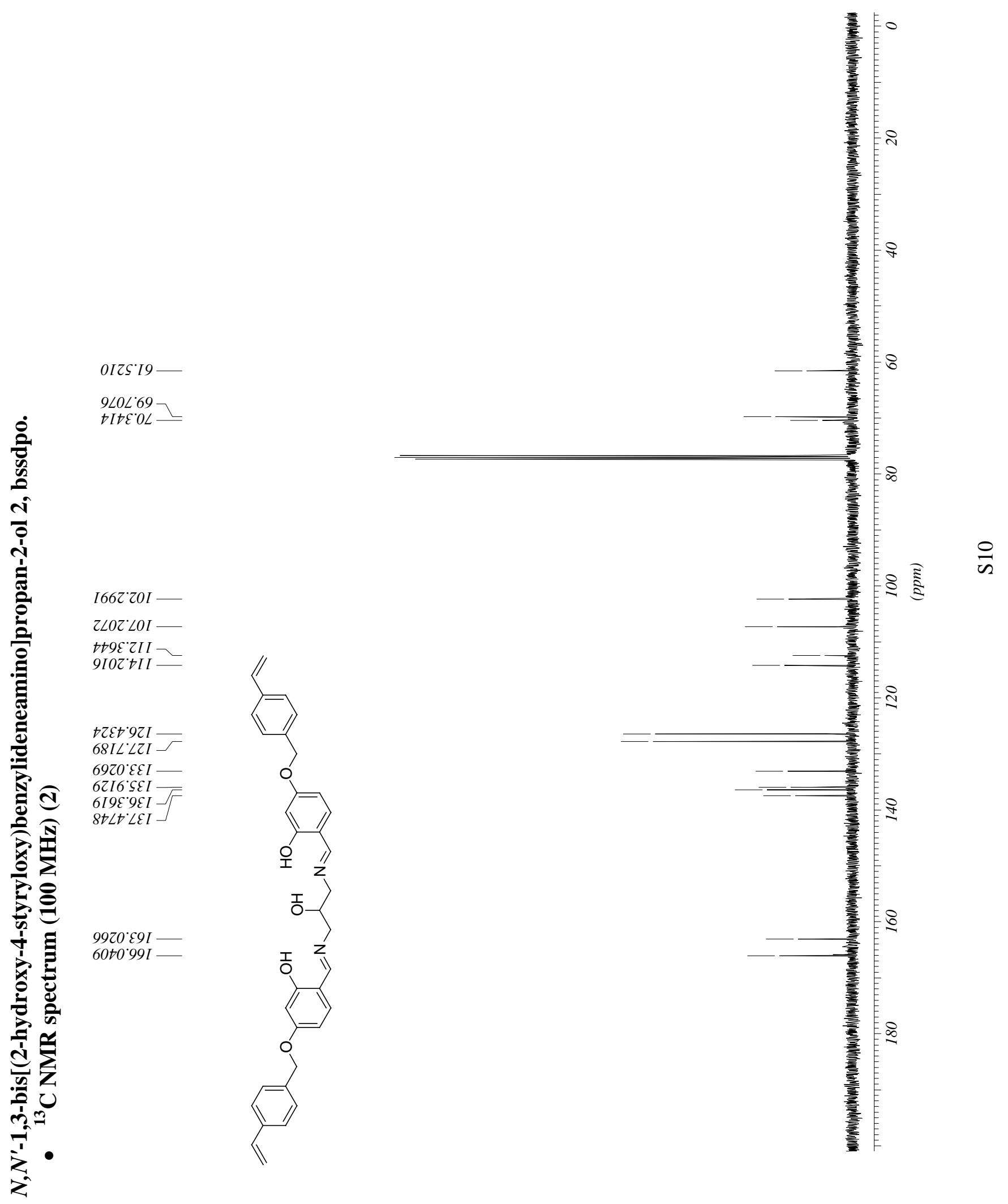



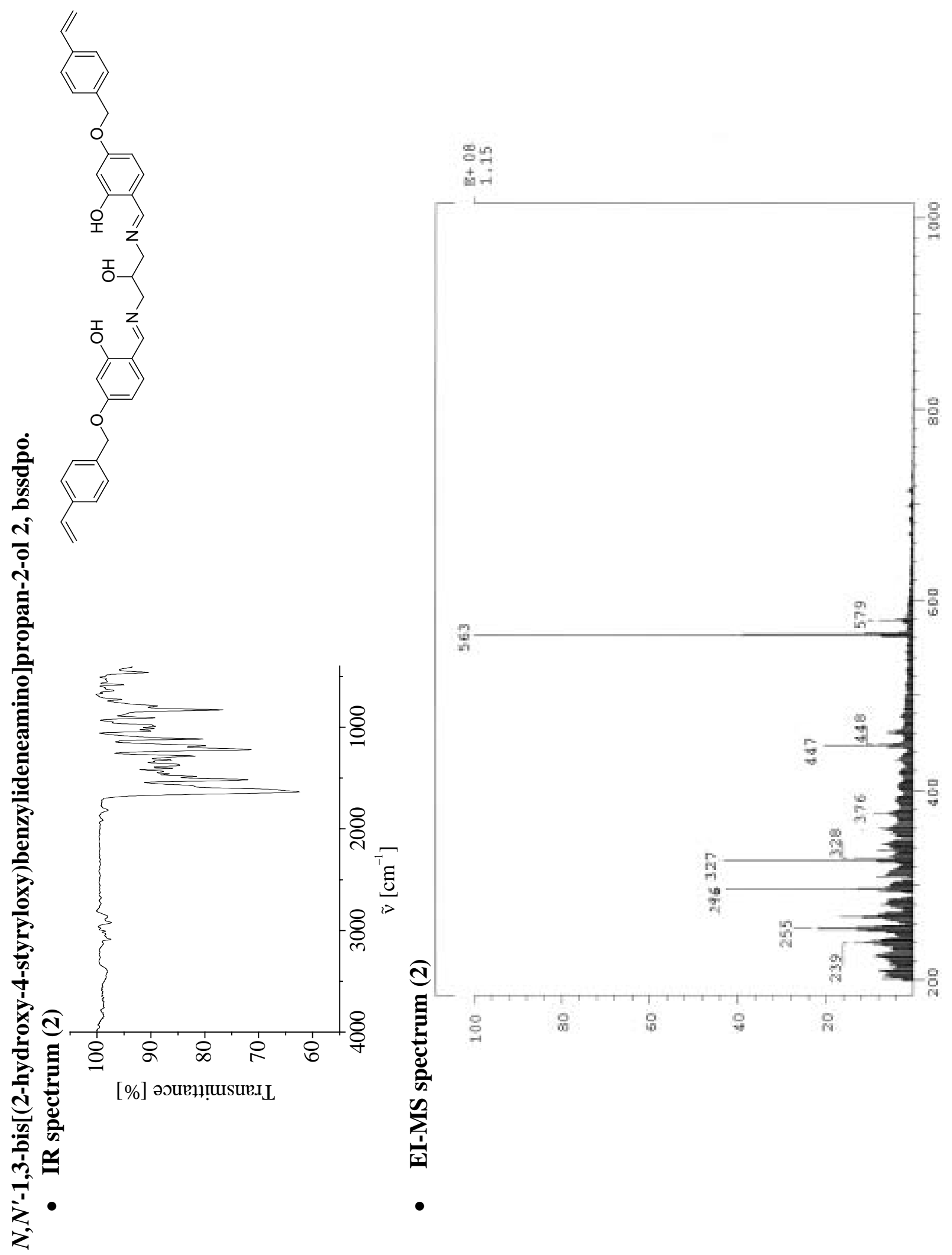

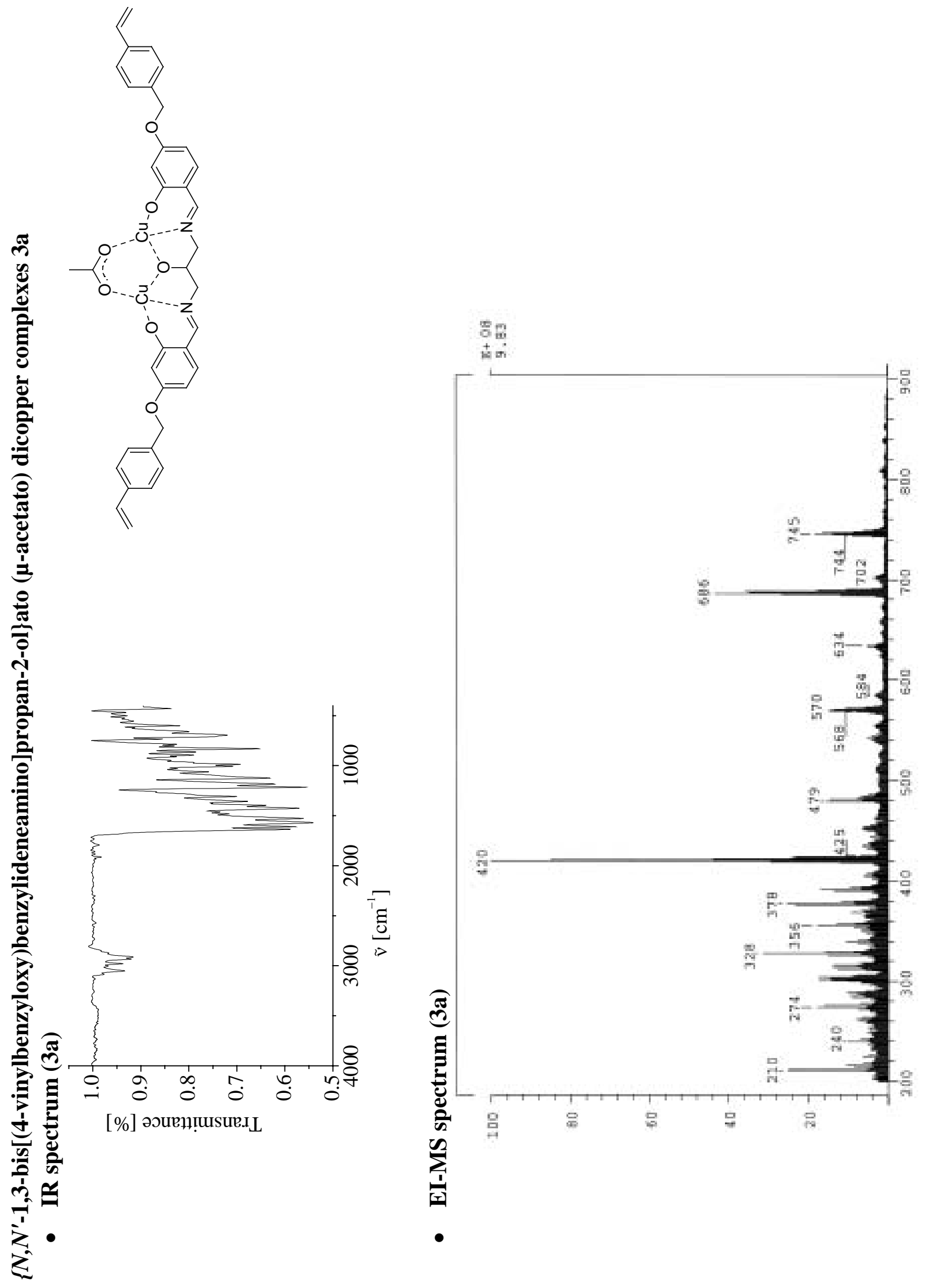

$\frac{N}{n}$ 

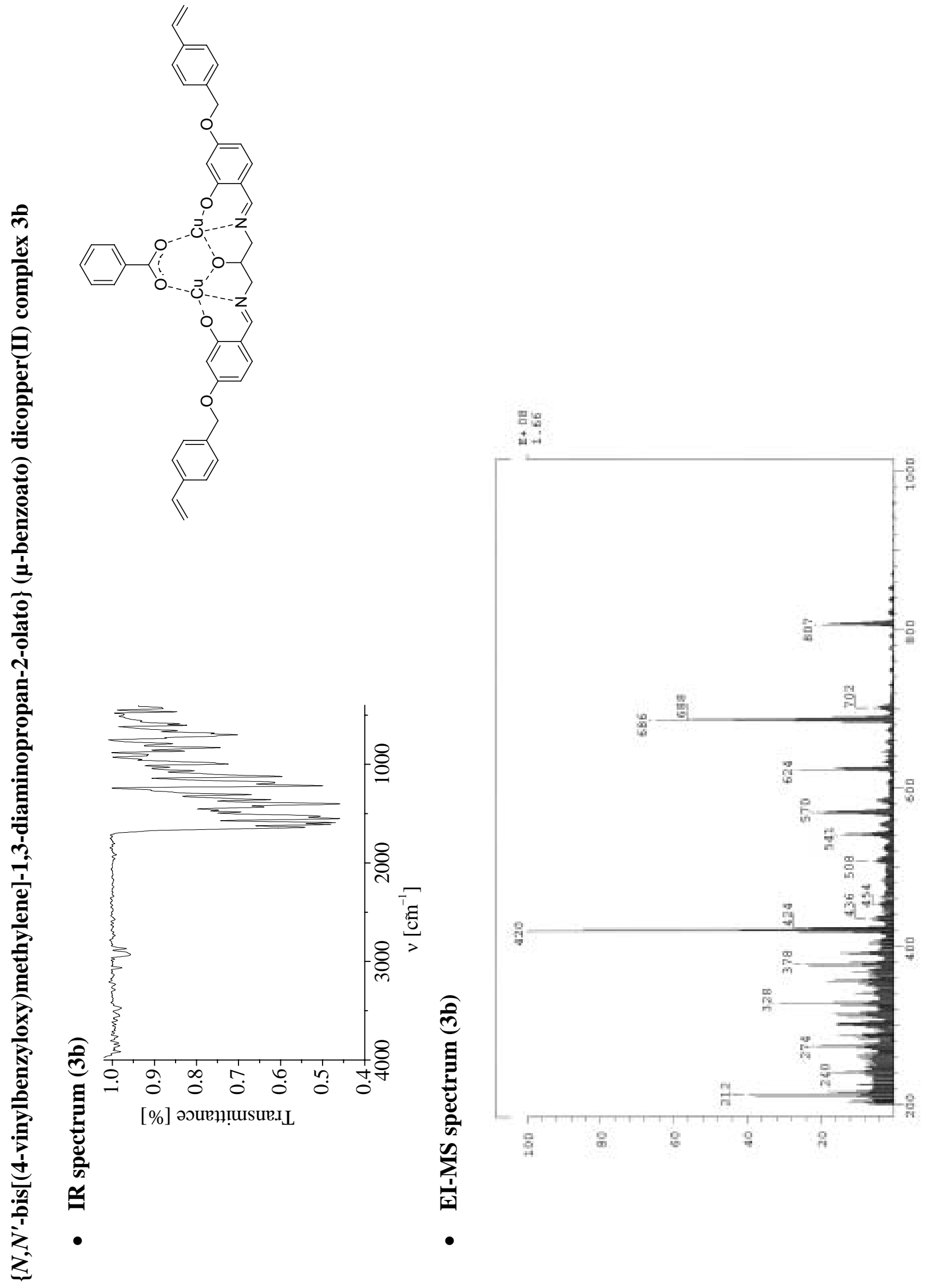

$\frac{n}{n}$ 